\title{
Epidemiologia dos Transtornos Mentais Comuns entre mulheres na zona rural de Rio Grande, RS, Brasil
}

\author{
Epidemiology of Common Mental Disorders among women \\ in the rural zones of Rio Grande, RS, Brazil
}

Pedro San Martin Soares (https://orcid.org/0000-0001-8974-7503) ${ }^{1}$

Rodrigo Dalke Meucci (https://orcid.org/0000-0002-8941-3850) ${ }^{1}$

${ }^{1}$ Faculdade de Medicina, Universidade Federal do Rio Grande. R. Gen. Osório s/n, Centro. 96200-400 Rio Grande RS Brasil. pedrosmsoares@ hotmail.com

\begin{abstract}
The scope of this study is to describe the prevalence and the factors associated with common mental disorders in women aged 18 to 49, residents in the rural zone of the municipality of Rio Grande-RS. A cross-sectional study was carried out with data collection conducted in 2017. A systematic sampling process of the residences of the rural area of the municipality was utilized. The General Health Questionnaire-12 (GHQ12) was used to track common mental disorders. Crude and adjusted Poisson regression was used to estimate the prevalence ratios $(P R)$ and respective 95\% confidence intervals. The overall prevalence of Common Mental Disorders was 36.4\%, being more common among women with lower levels of education, who had had an abortion and were smokers. In quantifying the occurrence and factors associated with common mental disorders in a population of women in a rural area, this study provides an original scientific contribution on the burden of this important outcome in a population that has not been studied in epidemiological surveys. The results can contribute to the health services by identifying the women most susceptible to this outcome, thus, improving the adequacy of the services offered to the health needs of rural residents.
\end{abstract}

Key words Mental health, Women, Rural population, Epidemiology
Resumo Este estudo tem por objetivo descrever a prevalência e os fatores associados aos transtornos mentais comuns em mulheres de 18-49 anos, residentes da zona rural do município de Rio Grande-RS. Foi realizado um estudo transversal com a coleta de dados realizada no ano de 2017. Foi utilizado um processo de amostragem sistemático dos domicílios da área rural do município. Para o rastreamento dos transtornos mentais comuns utilizou-se o General Health Questionnaire-12 (GHQ-12). Foi utilizada a regressão de Poisson bruta e ajustada para estimar as razões de prevalências $(R P)$ e os respectivos intervalos de confiança de $95 \%$. A prevalência geral de Transtornos Mentais Comuns foi de 36,4\%, sendo mais comum entre as mulheres com menor escolaridade, as que sofreram aborto e as tabagistas. Ao quantificar a ocorrência e os fatores associados aos transtornos mentais comuns numa população de mulheres de uma área rural, o presente estudo traz uma contribuição científica original sobre a carga deste importante desfecho numa população pouco estudada em inquéritos epidemiológicos. Os resultados podem contribuir com os serviços de saúde através da identificação das mulheres mais suscetiveis a este desfecho, melhorando deste modo a adequação dos serviços oferecidos às necessidades em saúde de moradores de áreas rurais.

Palavras-chave Saúde mental, Mulheres, População rural, Epidemiologia 


\section{Introdução}

Os Transtornos Mentais Comuns (TMC) são caracterizados por sintomas de depressão não psicótica, ansiedade e sintomas somatoformes (sintomas físicos relacionados a fatores psicológicos), em intensidade suficiente para interferir em atividades diárias, mas que não necessariamente preenchem os critérios formais da Classificação internacional de doenças (CID-10) ou do Manual diagnóstico e estatístico dos transtornos mentais (DSM-V). Seus principais sintomas são insônia, fadiga, esquecimento, irritabilidade, dificuldade de concentração, queixas somáticas e sentimento de inutilidade ${ }^{1}$. Diante do impacto dos TMC à saúde pública, foram criados diversos instrumentos para sua triagem. Entre os mais utilizados estão o Clinical Interview Schedule - Revised (CIS-R), o General Health Questionnaire (GHQ-12) e o Self Reporting Questionnare (SRQ-20), sendo este último o mais utilizado nos estudos brasileiros.

A elevada prevalência de TMC na população se mostrou uma característica mundial, variando de $14,7 \%$ a $21,8 \%$, sendo sua ocorrência maior nas mulheres, em comparação aos homens ${ }^{2,3}$. No Brasil, estudos de base populacional realizados em áreas urbanas evidenciaram prevalência de TMC em mulheres variando de $22,1 \%$ a $34,2 \%{ }^{4-}$ ${ }^{8}$. Em áreas rurais, apesar da relativa escassez de estudos nacionais, a prevalência de TMC em mulheres variou de $23,3 \%$ a $66,9 \% \%^{9-12}$.

A literatura aponta que, tanto no contexto rural quanto no urbano, a maior prevalência de TMC nas mulheres estaria relacionada a baixa escolaridade ${ }^{4,13,14}$, baixa renda ${ }^{4,14}$ e viver sem companheiro ${ }^{4,14}$. Além disso, são relatados outros fatores de risco, como cor da pele negra ou parda, ter filhos, não realizar atividades regulares de lazer, menopausa, já ter tido aborto e ter sofrido alguma agressão física ${ }^{13}$.

Além dos fatores sociais, as mulheres apresentariam uma vulnerabilidade biológica aos sintomas dos TMCs, especialmente os associados ao período reprodutivo. O papel do estrógeno na modulação do humor explicaria, parcialmente, a maior prevalência dos transtornos do humor e de ansiedade na mulher, da menarca à menopausa. Também têm sido associadas ao humor depressivo as situações de variação hormonal, como o período pré-menstrual, o puerpério, a menopausa, além de uso de contraceptivos orais e a terapia de reposição hormonal ${ }^{15}$.

A maioria dos estudos sobre TMC em mulheres foram conduzidos em áreas urbanas e em ci- dades de grande porte. Há poucos estudos sobre mulheres residentes em pequenos municípios, sobretudo em áreas rurais brasileiras. Em virtude da elevada ocorrência de TMC nessa população, que somada às características estressoras do ambiente rural, como longas distâncias, tendência a viver em isolamento, dificuldade em desenvolver outra atividade laboral ou de expressão cultural (trabalho exclusivo na lavoura), declínio da economia agrícola, renda irregular e desvantagens financeira e educacional, se fazem necessários estudos nesse cenário ${ }^{16}$.

Nesse sentido, a fim de contribuir para o avanço das pesquisas em saúde mental em mulheres da zona rural, este estudo tem por objetivo descrever a prevalência e os fatores associados aos transtornos mentais comuns em mulheres de 18-49 anos, residentes da zona rural do município de Rio Grande-RS.

\section{Metodologia}

Este estudo é resultado do consórcio de pesquisa do mestrado em Saúde Pública da Universidade Federal do Rio Grande denominado "Saúde da população rural Rio-Grandina”. Este consórcio contempla mulheres em idade fértil ( 15 a 49 anos), porém o presente estudo se detém nas mulheres de 18-49 anos. Constitui-se em um estudo transversal de base populacional na zona rural do município de Rio Grande, Rio Grande do Sul, Brasil, realizado entre os meses de abril e outubro do ano de 2017.

Para o estudo da prevalência dos transtornos mentais comuns foram considerados os seguintes paramêtros: nivel de confiança de $95 \%$, prevalência de $35 \%$ na população, uma margem de erro de 3 pontos percentuais e acréscimo de $10 \%$ para perdas. Para o estudo dos fatores associados foram utilizados os paramêtros: nivel de confiança de $95 \%$, poder de $80 \%$, razão de prevalência de 2,0, prevalência de TMC entre os não expostos, o qual variou de $15 \%$ a $25 \%$, razão de não expostos/expostos de 3:2 a 9:1, mais acréscimo de $15 \%$ para controle de fatores de confusão. Deste modo, o maior tamanho de amostra necessária seria de 778 mulheres.

A zona rural do município de Rio Grande é constituída por 24 setores censitários com cerca de 8.500 habitantes distribuídos em aproximadamente 2.700 domicílios permanentemente habitados. Para a seleção dos participantes utilizou-se o processo por amostragem sistemática, de modo a selecionar $80 \%$ dos domicílios. Este processo 
foi realizado através do sorteio de um número entre " 1 " e " 5 ", sendo que o sorteado correspondeu ao domicílio considerado pulo. Por exemplo, no caso do número " 3 " ter sido sorteado, todo domicílio de número " 3 ", de uma sequência de cinco domicílios, não era amostrado, ou seja, era pulado. Este procedimento garantiu que fossem amostrados quatro em cada cinco domicílios.

O critério de inclusão deste estudo foi mulheres com idade de 18 a 49 anos residentes na zona rural do município de Rio Grande-RS. Foram consideradas inelegíveis aquelas com incapacidade de comunicação verbal, cognitiva ou mental, observada pelo supervisor do trabalho de campo e pelas institucionalizadas (hospitalizadas ou encarceradas).

Foram coletadas as seguintes variáveis: idade (em anos completos no momento da entrevista), escolaridade (em anos completos), situação conjugal (casada/com companheiro, e as mulheres que vivam sozinhas foram classificadas como solteiras ou separadas/divorciadas/viúvas), situação de trabalho, renda familiar (valor recebido por todos os moradores do domicílio no mês imediatamente anterior à entrevista), idade que engravidou pela primeira vez, número de filhos nascidos vivos, e ocorrência de aborto espontâneo ou provocado em algum momento no passado.

O número de atividades sociais foi caracterizado como a soma das respostas afirmativas das seguintes perguntas relativas aos últimos 30 dias: "Foi a missa ou culto na igreja?"; "Foi a uma festa na comunidade?"; "Foi a uma festa da família?"; "Foi a algum baile?" " "Viajou para outra cidade?". A variável atividade física foi caracterizada como a presença de pelo menos uma dessas atividades nos últimos sete dias por no mínimo $30 \mathrm{~min} / \mathrm{dia}$ : "Caminhar", "carregar pesos leves", "carregar objetos pesados", "trabalhar com enxada", "andar de bicicleta" e "nadar". O índice de massa corporal (IMC) foi calculado a partir do peso e da altura autorrelatados e utilizados os pontos de corte propostos pela OMS, no qual considera-se baixo peso o IMC menor que 18,5 , peso adequado o intervalo entre $\geq 18,5$ e 25 , sobrepeso entre $\geq 25$ e $<30$ e obesidade IMC $\geq 25 \mathrm{~kg} / \mathrm{m}^{2}{ }^{17}$. As categorias "baixo peso" e "peso adequado" foram agregadas na operacionalização, devido às participantes com IMC menor que $18,5 \mathrm{Kg} / \mathrm{m}^{2}$ representarem menos de $2 \%$ da amostra. Também foram avaliadas as variáveis uso de álcool nos últimos sete dias, tabagismo (pelo menos um cigarro por dia no último mês) e consulta ao médico nos últimos 12 meses.

Para avaliar a presença de Transtornos Mentais comuns foi utilizado o General Health Ques- tionnaire-12 (GHQ-12). Esse instrumento avalia a presença de Transtornos Mentais Comuns nas últimas duas semanas através de 12 itens, sendo seu ponto de corte $\geq 3$ pontos. Sua validação na população brasileira possui uma sensibilidade de $85 \%$ e especificidade de $79 \%{ }^{18}$. O GHQ-12 se mostrou apropriado para estudos na população geral, além de avaliar a presença de TMC de forma semelhante ao SRQ-20 $0^{18,19}$.

A coleta de dados foi realizada no domicílio por entrevistadoras treinadas e selecionadas utilizando questionário contendo informações sociodemográficas, reprodutivas, comportamentais e de saúde mental aplicado em forma de entrevista individual. Utilizou-se um questionário eletrônico com o registro dos dados em tablets através do aplicativo móvel do software RedCap (Research Eletronic Data Capture) ${ }^{20}$.

Para a análise dos dados foi utilizado o programa Stata/IC ${ }^{\circledR} 13$, sendo inicialmente realizadas análises descritivas das variáveis coletadas. Em seguida foi calculada a prevalência dos transtornos mentais comuns e seu respectivo intervalo de confiaça de $95 \%$. Análises brutas e ajustadas foram realizadas através regressão de Poisson com variância robusta para estimar as razões de prevalências (RP) e seus intervalos de confiança de $95 \%$.

Para a análise ajustada dos fatores associados aos Transtornos Mentais Comuns, empregou-se um modelo hierárquico em quatro níveis para ajuste de possíveis fatores de confundimento, embasado na revisão da literatura, no qual as variáveis sociodemográficas, consideradas distais, determinam renda e trabalho, que por sua vez, determinam história reprodutiva e aspectos comportamentais e nutricionais ${ }^{21}$. Neste modelo, as variáveis, situadas em um nível hierarquicamente superior ao daquela em questão, foram consideradas como potenciais confundidores na relação com o desfecho em estudo, enquanto as situadas em níveis inferiores foram consideradas como potenciais mediadores da associação ${ }^{21}$.

Desse modo, utilizou-se, no primeiro nível, as variáveis socioeconômicas, no segundo, as demográficas, no terceiro, as obstétricas, e, no último, as comportamentais. Foram mantidas na análise todas as associações que apresentaram p de significância menor ou igual a 0,20 no teste Wald de heterogeneidade ou tendência linear. Foram consideradas significativas apenas aquelas cujo $\mathrm{p}$ de significância foram menor a 0,05 .

De acordo com a resolução 466/12, este protocolo de pesquisa, considerado de risco mínimo, foi aprovado pelo Comitê de Ética em Pesquisa 
na Área da Saúde (CEPAS), da Universidade Federal do Rio Grande. Além disso, garantiu-se a confidencialidade dos dados, a participação voluntária e a possibilidade de deixar o estudo a qualquer momento, sem necessidade de justificativa. Todos os participantes assinaram o Termo de Consentimento Livre e Esclarecido (TCLE) antes de o questionário ser aplicado.

\section{Resultados}

Dentre as 1.199 mulheres amostradas para o estudo geral, foram entrevistadas 1.079 (10\% de perdas e recusas), das quais 996 tinham de 18 a 49 anos.

A Tabela 1 descreve as características da população estudada. Um terço das mulheres tinha idade entre 18 a 29 anos, mais da metade possuía até oito anos de estudo, $83,2 \%$ eram casadas ou viviam com companheiro e $37,2 \%$ estavam tra-

Tabela 1. Caracteristicas gerais das mulheres da zona rural de Rio Grande, Rio Grande do Sul, Brasil, 2017 $(\mathrm{N}=996)$.

\begin{tabular}{lr}
\hline \multicolumn{1}{c}{ Variáveis } & $\begin{array}{c}\text { Frequência } \\
(\%)\end{array}$ \\
\hline Idade $(\mathrm{n}=996)$ & $349(35,0)$ \\
18 a 29 anos & $333(33,5)$ \\
30 a 39 anos & $314(31,5)$ \\
40 a 49 anos & \\
Escolaridade $(\mathrm{n}=995)$ & $219(22,0)$ \\
0 a 4 anos & $346(34,8)$ \\
5 a 8 anos & $102(10,3)$ \\
9 a 11 anos & $328(32,9)$ \\
12 anos ou mais & \\
Situação conjugal (n =996) & $122(12,2)$ \\
Solteira & $828(83,2)$ \\
Casada ou com companheiro & $46(4,6)$ \\
Separada, divorciada ou viúva & \\
Trabalho (n = 995) & $136(13,7)$ \\
Com carteira assinada & $234(23,5)$ \\
Sem carteira assinada & $625(62,8)$ \\
Não trabalha & \\
Idade que engravidou a primeira vez & \\
(n = 990) & $175(17,7)$ \\
Nunca engravidou & $414(41,8)$ \\
27 anos ou mais & $160(16,2)$ \\
22 a 26 anos & \\
17 a 21 anos & \\
13 a 16 anos & \\
\hline & \\
&
\end{tabular}

balhando. Ainda, nessa mesma tabela, é possivel verificar que $41,8 \%$ das mulheres engravidaram pela primeira vez na faixa etária entre 17 e 21 anos, $31,7 \%$ possuiam um filho e $15,7 \%$ relataram já ter tido um aborto. Em relação às variáveis comportamentais, $39 \%$ realizaram apenas uma atividade social no último mês, $65,5 \%$ realizaram alguma atividade fisica nos últimos sete dias, 9,7\% consumiram alguma bebida de álcool na última semana, $15,4 \%$ eram tabagistas e $70,2 \%$ relataram ter consultado um médico nos últimos

Tabela 1. Caracteristicas gerais das mulheres da zona rural de Rio Grande, Rio Grande do Sul, Brasil, 2017 $(\mathrm{N}=996)$.

\begin{tabular}{|c|c|}
\hline Variáveis & $\begin{array}{l}\text { Frequência } \\
(\%)\end{array}$ \\
\hline \multicolumn{2}{|l|}{ Número de filhos $(\mathrm{n}=995)$} \\
\hline Nenhum & $171(17,2)$ \\
\hline Um & $315(31,7)$ \\
\hline Dois & $299(30,0)$ \\
\hline Três ou mais & $210(21,1)$ \\
\hline \multicolumn{2}{|c|}{ Já teve algum aborto $(\mathrm{n}=994)$} \\
\hline Sim & $156(15,7)$ \\
\hline Não & $838(84,3)$ \\
\hline \multicolumn{2}{|c|}{$\begin{array}{l}\text { Atividade social nos últimos } 30 \text { dias } \\
(\mathrm{n}=996)\end{array}$} \\
\hline Duas ou mais & $373(37,5)$ \\
\hline Uma & $388(39,0)$ \\
\hline Nenhuma & $235(23,5)$ \\
\hline \multicolumn{2}{|c|}{$\begin{array}{l}\text { Atividade física (na última semana) } \\
(\mathrm{n}=992)\end{array}$} \\
\hline Não & $342(34,5)$ \\
\hline Sim & $650(65,5)$ \\
\hline \multicolumn{2}{|l|}{$\operatorname{IMC}(\mathrm{n}=973)$} \\
\hline Até 24,99 & $300(30,8)$ \\
\hline De 25 a 29,99 & $334(34,3)$ \\
\hline 30 ou mais & $339(34,9)$ \\
\hline \multicolumn{2}{|l|}{ Tabagismo $(\mathrm{n}=996)$} \\
\hline $\operatorname{Sim}$ & $153(15,4)$ \\
\hline Não & $843(84,6)$ \\
\hline \multicolumn{2}{|c|}{ Álcool (na última semana) $(\mathrm{n}=996)$} \\
\hline Sim & $97(9,7)$ \\
\hline Não & $899(90,3)$ \\
\hline \multicolumn{2}{|c|}{$\begin{array}{l}\text { Consultou médico nos últimos } 12 \\
\text { meses }(\mathrm{n}=994)\end{array}$} \\
\hline Sim & $698(70,2)$ \\
\hline Não & $296(29,8)$ \\
\hline \multicolumn{2}{|c|}{ Transtornos Mentais Comuns $(\mathrm{n}=993)$} \\
\hline Sim & $361(36,4)$ \\
\hline Não & $632(63,6)$ \\
\hline
\end{tabular}


Tabela 2. Análise bruta e ajustada para Transtornos Mentais Comuns de acordo com as características analisadas em mulheres da zona rural de Rio Grande, Rio Grande do Sul, Brasil, 2017 (N = 996).

\begin{tabular}{|c|c|c|c|c|}
\hline & \multirow{2}{*}{ Variáveis } & \multirow{2}{*}{$\begin{array}{c}\text { Frequência } \\
\mathbf{n}(\%)\end{array}$} & \multirow{2}{*}{$\begin{array}{l}\text { Análise Bruta } \\
\text { RP }^{\|}(\mathrm{IC} 95 \%)^{\dagger}\end{array}$} & \multirow{2}{*}{$\begin{array}{c}\text { Análise Ajustada } \\
\text { RP }^{\|}(\mathrm{IC} 95 \%)^{\dagger}\end{array}$} \\
\hline & & & & \\
\hline \multirow[t]{13}{*}{$1^{\circ}$ Nivel } & Idade & & $0,014^{*}$ & $0,420^{*}$ \\
\hline & 18 a 29 anos & $110(31,5 \%)$ & 1,00 & 1,00 \\
\hline & 30 a 39 anos & $116(35,1 \%)$ & $1,11(0,86-1,44)$ & $1,06(0,81-1,38)$ \\
\hline & 40 a 49 anos & $135(43,1 \%)$ & $1,36(1,06-1,76)$ & $1,12(0,85-1,47)$ \\
\hline & Escolaridade & & $<0,001^{\star}$ & $0,004^{*}$ \\
\hline & 0 a 4 anos & $105(48,2 \%)$ & $1,74(1,31-2,30)$ & $1,56(1,15-2,14)$ \\
\hline & 5 a 8 anos & $133(38,4 \%)$ & $1,39(1,06-1,81)$ & $1,30(0,98-1,74)$ \\
\hline & 9 a 11 anos & $32(32,0 \%)$ & $1,15(0,77-1,73)$ & $1,11(0,73-1,70)$ \\
\hline & 12 anos ou mais & $91(27,7 \%)$ & 1,00 & 1,00 \\
\hline & Situação conjugal & & $0,014^{* *}$ & $0,347^{\star *}$ \\
\hline & Solteira & $29(23,8 \%)$ & 1,00 & 1,00 \\
\hline & Casada ou com companheiro & $308(37,3 \%)$ & $1,57(1,07-2,30)$ & $1,30(0,84-2,01)$ \\
\hline & Separada, divorciada ou viúva & $24(52,2 \%)$ & $2,19(1,28-3,77)$ & $1,57(0,84-2,93)$ \\
\hline \multirow[t]{10}{*}{$2^{\circ}$ Nivel } & Trabalho & & $0,020^{\star *}$ & $0,319^{* *}$ \\
\hline & Com carteira assinada & $31(22,8 \%)$ & 1,00 & 1,00 \\
\hline & Sem carteira assinada & $89(38,2 \%)$ & $1,68(1,11-2,52)$ & $1,37(0,89-2,10)$ \\
\hline & Não trabalha & $241(38,7 \%)$ & $1,70(1,17-2,47)$ & $1,35(0,89-2,02)$ \\
\hline & Renda (quintil) $(\mathrm{n}=927)$ & & $0,001^{*}$ & $0,065^{\star}$ \\
\hline & Primeiro (mais pobre) & $81(43,3 \%)$ & $1,81(1,24-2,62)$ & $1,48(1,01-2,19)$ \\
\hline & Segundo & $75(40,3 \%)$ & $1,68(1,15-2,45)$ & $1,38(0,93-2,05)$ \\
\hline & Terceiro & $79(39,1 \%)$ & $1,63(1,12-2,37)$ & $1,40(0,95-2,06)$ \\
\hline & Quarto & $58(33,1 \%)$ & $1,38(0,93-2,05)$ & $1,29(0,86-1,92)$ \\
\hline & Quinto (mais rico) & $42(24,0 \%)$ & 1,00 & 1,00 \\
\hline \multirow[t]{14}{*}{$3^{\circ}$ Nivel } & Idade que engravidou a primeira vez $(\mathrm{n}=990)$ & & $0,019^{* *}$ & $0,420^{* *}$ \\
\hline & Nunca engravidou & $37(23,7 \%)$ & 1,00 & 1,00 \\
\hline & 27 anos ou mais & $25(29,4 \%)$ & $1,24(0,75-2,06)$ & $1,08(0,62-1,87)$ \\
\hline & 22 a 26 anos & $76(43,4 \%)$ & $1,83(1,24-2,71)$ & $1,45(0,94-2,25)$ \\
\hline & 17 a 21 anos & $154(37,4 \%)$ & $1,58(1,10-2,26)$ & $1,22(0,81-1,83)$ \\
\hline & 13 a 16 anos & $67(42,1 \%)$ & $1,78(1,19-2,65)$ & $1,35(0,86-2,11)$ \\
\hline & Número de filhos & & $0,002^{\star}$ & $0,719^{\star}$ \\
\hline & Nenhum & $45(26,3 \%)$ & 1,00 & 1,00 \\
\hline & Um & $110(34,9 \%)$ & $1,33(0,94-1,88)$ & $0,76(0,35-1,65)$ \\
\hline & Dois & $108(36,2 \%)$ & $1,38(0,97-1,95)$ & $0,73(0,33-1,58)$ \\
\hline & Três ou mais & $97(46,6 \%)$ & $1,77(1,24-2,52)$ & $0,84(0,38-1,85)$ \\
\hline & Já teve algum aborto & & $0,001^{* *}$ & $0,008^{* *}$ \\
\hline & Não & $281(33,6 \%)$ & 1,00 & 1,00 \\
\hline & Sim & $79(50,9 \%)$ & $1,52(1,18-1,95)$ & $1,39(1,07-1,83)$ \\
\hline
\end{tabular}


Tabela 2. Análise bruta e ajustada para Transtornos Mentais Comuns de acordo com as características analisadas em mulheres da zona rural de Rio Grande, Rio Grande do Sul, Brasil, 2017 ( N = 996).

\begin{tabular}{|c|c|c|c|c|}
\hline & \multirow{2}{*}{ Variáveis } & \multirow{2}{*}{$\begin{array}{c}\text { Frequência } \\
\text { n (\%) }\end{array}$} & \multirow{2}{*}{$\begin{array}{l}\text { Análise Bruta } \\
\text { RP }^{\|}(\text {IC95\% })^{\dagger}\end{array}$} & \multirow{2}{*}{$\begin{array}{c}\text { Análise Ajustada } \\
\text { RP }^{\|}(\mathrm{IC} 95 \%)^{\dagger}\end{array}$} \\
\hline & & & & \\
\hline \multirow[t]{21}{*}{$4^{\circ}$ Nivel } & Atividade social nos últimos & & $0,095^{\star}$ & $0,242^{\star}$ \\
\hline & 30 dias & & & \\
\hline & Nenhuma & $93(39,6)$ & $1,24(0,95-1,63)$ & $1,21(0,91-1,62)$ \\
\hline & Uma & $150(38,8)$ & $1,21(0,96-1,55)$ & $1,11(0,86-1,44)$ \\
\hline & Duas ou mais & $118(31,8)$ & 1,00 & 1,00 \\
\hline & Atividade física (na última semana) & & $0,540^{\star *}$ & $0,925^{* *}$ \\
\hline & Não & $130(38,1 \%)$ & 1,00 & 1,00 \\
\hline & Sim & $231(35,7 \%)$ & $0,94(0,75-1,16)$ & $1,01(0,79-1,26)$ \\
\hline & IMC & & $0,277^{\star \star}$ & $0,747^{\star *}$ \\
\hline & Até 24,99 & $99(33,1 \%)$ & 1,00 & 1,00 \\
\hline & De 25 a 29,99 & $118(35,3 \%)$ & $1,07(0,82-1,40)$ & $1,48(1,05-2,19)$ \\
\hline & 30 ou mais & $187(40,5 \%)$ & $1,22(0,95-1,59)$ & $1,38(0,93-2,06)$ \\
\hline & Álcool (na última semana) & & $0,963^{\star *}$ & $0,828^{* *}$ \\
\hline & Não & $326(36,4 \%)$ & 1,00 & 1,00 \\
\hline & Sim & $35(36,1 \%)$ & $0,99(0,70-1,41)$ & $1,04(0,72-1,50)$ \\
\hline & Tabagismo & & $0,010^{\star *}$ & $0,046^{* *}$ \\
\hline & Não & $288(34,2 \%)$ & 1,00 & 1,00 \\
\hline & $\operatorname{Sim}$ & $73(48,0 \%)$ & $1,40(1,09-1,81)$ & $1,32(1,01-1,74)$ \\
\hline & Consultou médico nos últimos 12 meses & & $0,055^{\star *}$ & $0,090^{* *}$ \\
\hline & Não & $91(30,7)$ & 1,00 & 1,00 \\
\hline & Sim & $270(38,8)$ & $1,26(0,99-1,60)$ & $1,25(0,97-1,61)$ \\
\hline
\end{tabular}

\section{Discussão}

Este estudo observou que uma em cada três mulheres da zona rural do município de Rio Grande-RS apresenta TMC. As mulheres com menor escolaridade, as que sofreram algum aborto durante a vida e as tabagistas apresentaram maior risco para TMC após ajuste para fatores de confusão.

Estudos demonstram altos índices de prevalência geral de TMC na população adulta brasileira, variando entre $20 \%$ e $56 \%$, e acometendo principalmente mulheres e trabalhadores ${ }^{22}$. A prevalência de TMC encontrada no presente estudo, medida pelo GHQ-12, foi semelhante à relatada na literatura para mulheres de faixa etária similar residentes em áreas rurais de outras regiões do Brasil. Em dois estudos realizados com o "Self Reporting Questionnaire" (SRQ-20), aplicado em mulheres de 15 a 49 anos, a prevalência de TMC no último mês variou entre 40,2\% (IC95\%: 37,3 - 43,1) e 35,7\% (IC95\%: 30,1\% 41,4\%) na Zona da Mata de Pernambuco e em Uberaba (MG), respectivamente ${ }^{23,24}$. Em com- paração com achados relatados em mulheres de intervalo de idades semelhante em áreas urbanas, um estudo encontrou uma prevalência de 35,8\% (IC95\%: 33,2 - 38,4) em Feira de Santana (Bahia) ${ }^{25}$ e outro uma inferior, 27,3\% (IC95\%: 24,2 - 30,3), no município de São Paulo ${ }^{23}$.

As altas prevalências de TMC em ambos os contextos, rural e urbano, podem ser explicados pelo fato da maior percepção das mulheres com a própria saúde, pela desigualdade entre os sexos, pela maior responsabilidade social das mulheres em relação aos homens e a pela desvalorização do trabalho feminino ${ }^{3}$. $\mathrm{Na}$ zona rural, além do valores conservadores serem mais presentes em relação a zona urbana, características como altas taxas de pobreza, isolamento e falta de perspectiva da melhoria das condições de vida podem contribuir para esses resultados ${ }^{26}$.

A relação entre TMC e baixa escolaridade é relatada em pesquisas de base populacional realizadas com mulheres da Índia, Paraguai e Brasil $^{27-29}$. A escolaridade possui uma relação direta com a saúde mental, pois influencia nas escolhas na vida, nas aspirações, na autoestima e na aqui- 
sição de novos conhecimentos, os quais podem contribuir para atitudes e comportamentos mais saudáveis ${ }^{30}$. A escolaridade também permite qualificar os indivíduos para certas ocupações e interfere nas suas condições socioeconômicas futuras e inserção na estrutura ocupacional ${ }^{30}$. A falta de oportunidade de educação nas áreas rurais é responsável pela existência de uma lacuna entre mulheres urbanas e rurais, o que agravaria a situação destas últimas, principalmente na redução da capacidade para lidar com situações estressoras de maneira adequada, reduzindo a disposição para suportar acontecimentos adversos e frustrantes ${ }^{31}$.

Em relação à associação entre aborto e TMC, o presente estudo encontrou resultados semelhantes ao de outros estudos que fizeram essa associação $0^{13,32,33}$. A relação entre saúde mental e aborto foi evidenciada pelo estudo longitudinal realizado com 500 mulheres na Nova Zelândia, que avaliou a saúde mental antes e depois do aborto. Após a análise ajustada, considerando possíveis fatores de confusão sugeridos na literatura, o aborto esteve associado ao aumento consistente no risco de problemas de saúde mental ${ }^{32}$. $\mathrm{Na}$ gestação, as mulheres criam fantasias sobre o bebê que esta por vir, que envolvem, principalmente, o sexo, o nome, a maneira como ele se movimenta no útero, e as características psicológicas que são a ele atribuídas. Portanto, independentemente do desejo pela gestação, o aborto interrompe um percurso biológico natural e esperado socialmente, fazendo com que a mulher se depare com a expectativa de não haver maternidade $^{34}$. A frustração decorrente da interrupção dessa expectativa explicaria a vulnerabilidade dessas mulheres para os TMC.

Estudos transversais de base populacional mostram que mulheres tabagistas apresentam maior prevalência de TMC em comparação às não tabagistas, porém, não esclarecem a temporalidade dessa associação ${ }^{27,35,36}$. Estudo longitudinal, realizado na Índia, com 2.094 mulheres, com idade entre 18 e 45 anos, constatou que tabagistas apresentaram uma incidência de TMC quatro vezes maior em relação às não fumantes ${ }^{37}$.

Há evidencias da atuação da nicotina, presente no tabaco, na desregulação no eixo hipotálamopituitária-adrenal e no aumento da ação do cortisol no cérebro ${ }^{38}$. Estas alterações fisiopatológicas são consideradas características dos sintomas depressivos $^{39}$, portanto, o tabagismo poderia ge- rar maior suscetibilidade para o desenvolvimento de TMC. Além disso, o tabaco funcionaria como uma "automedicação" para os sintomas do humor depressivo e ansiosos, presentes nos $\mathrm{TMC}^{40}$.

Deve-se ressaltar que este estudo possui um delineamento transversal, tendo exposição e desfecho avaliados no mesmo momento. Dessa forma, os resultados podem estar sujeitos à limitação da causalidade reversa, por não ser possível o estabelecimento da temporalidade. Outra limitação é a não inclusão das adolescentes de 15 a 17 anos, as quais fazem parte do grupo populacional das mulheres em idade fértil.

Considerando as vantagens do presente estudo, cabe destacar que se trata de uma pesquisa de base populacional com uma amostra representativa e com nível mínimo de perdas. Apesar da ampla produção científica sobre os TMC no Brasil, ainda são escassos os estudos de base populacional realizados na zona rural.

Em relação ao instrumento utilizado para o rastreamento de TMC, este estudo utilizou um que foi validado na população brasileira e empregado em outros países em desenvolvimento ${ }^{41-43}$.

A ocorrência de TMC na zona rural do município de Rio Grande é uma condição comum entre as mulheres avaliadas. Apesar de não ter identificado novos fatores de risco ou proteção, os achados são consistentes com os resultados relatados em estudos realizados em áreas urbanas.

É necessário, portanto, promover atividades de acolhimento em saúde mental, a fim de identificar e encaminhar os casos de TMC, com prioridade para mulheres com baixa escolaridade, que sofreram aborto e que são tabagistas. Intervenções em mulheres jovens se mostram necessárias, uma vez que estudos brasileiros com idosos residentes na zona rural evidenciam um aumento da prevalência de TMC com o avanço da idade $\mathrm{e}^{11,12}$. Este estudo destaca a importância da doença mental enquanto problema de saúde pública também na zona rural. A criação de programas de atendimento direcionados aos moradores rurais, visando o rastreamento e o diagnóstico precoce dos transtornos mentais comuns e a manutenção do tratamento, é ação importante, que deve ser fomentada pelas políticas locais e nacionais em saúde. Isso se torna ainda mais importante em regiões rurais com elevada cobertura da atenção básica, mas que dependem de encaminhamento aos serviços de atenção psicossocial localizados em áreas urbanas. 


\section{Colaboradores}

Atestamos também que PSM Soares e RD Meucci participaram da concepção e revisões que resultaram neste artigo.

\section{Referências}

1. Goldberg D, Huxley P. Common Mental Disorders. A Bio-social Model. London: Routledge; 1992.

2. Steel Z, Marnane C, Iranpour C, Chey T, Jackson JW, Patel V, Silove D. The global prevalence of common mental disorders: a systematic review and meta-analysis 1980-2013. Int J Epidemiol 2014; 43(2):476-493.

3. Fonseca MLG, Guimarães MBL, Vasconcelos EM. Sofrimento difuso e transtornos mentais comuns: uma revisão bibliográfica. Rev APS 2008; 11(3):285-294.

4. Rocha SV, Almeida MM, Araujo TM, Virtuoso Júnior JS. Prevalence of common mental disorders among the residents of urban areas in Feira de Santana, Bahia. Rev Bras Epidemiol 2010; 13(4):630-640.

5. Marin-Leon L, Oliveira HB, Barros MB, Dalgalarrondo P, Botega NJ. Social inequality and common mental disorders. Rev Bras Psiquiatr. 2007; 29(3):250-253.

6. Costa JSD, Menezes AMB, Olinto MTA, Gigante DP, Macedo S, Britto MAP, Fuchs SC. Prevalência de distúrbios psiquiátricos menores na cidade de Pelotas, RS. Revista brasileira de epidemiologia 2002; 5(2):164173.

7. Mendoza-Sassi RA, Béria JU. Gender differences in self-reported morbidity: evidence from a population -based study in southern Brazil. Cad Saude Publica 2007; 23(2):341-346.

8. Rodrigues-Neto JF, Figueiredo MFS, Faria AAS, Fagundes M. Transtornos mentais comuns e o uso de práticas de medicina complementar e alternativa: estudo de base populacional. J Bras Psiquiatr 2008; 57(4):233-239.

9. Costa AG, Ludermir AB. Transtornos mentais comuns e apoio social: estudo em comunidade rural da Zona da Mata de Pernambuco, Brasil. Cad Saude Publica 2005; 21(1):73-79.

10. Lima PJP. Avaliação de transtornos mentais comuns em comunicades rurais em Atibaia/SP-Brasil. Cadernos Brasileiros de Saúde Mental 2015; 7(15):101-121.

11. Pinto LLT, Rocha SV, Viana HPS, Rodrigues WKM, Vasconcelos LRC. Nível de atividade física habitual e transtornos mentais comuns entre idosos residentes em áreas rurais. Revista Brasileira de Geriatria e Gerontologia 2014; 17(4):819-828.

12. Silva PAS, Rocha SV, Santos LB, Santos CA, Amorim CR, Vilela ABA. Prevalência de transtornos mentais comuns e fatores associados entre idosos de um município do Brasil. Cien Saude Colet 2018; 23(2):639646.

13. Araujo TM, Almeida MG, Santana CR, Araújo EM, Pinho PS. Transtornos mentais comuns em mulheres: estudo comparativo entre donas-de-casa e trabalhadoras. $R$ Enferm UERJ 2006; 14(2):260-269.

14. Araújo TM, Pinho PS, Almeida MMG. Prevalência de transtornos mentais comuns em mulheres e sua relação com as características sociodemográficas e o trabalho doméstico. Revista Brasileira de Saúde Materno Infantil 2005; 5(3):337-348.

15. Andrade LHSG, Viana MC, Silveira CM. Epidemiologia dos transtornos psiquiátricos na mulher. Rev Psiquiatr Clin 2006; 33(2):43-54.

16. Winters C, Cudney S, Sullivan T. Expressions of depression in rural women with chronic illness. Rural and remote health 2010; 10(4):1533. 
17. World Health Organization (WHO). Defining the problem of overweight and obesity. Obesity: preventing and managing the global epidemic: report of a WHO Consultation. Geneva: WHO; 2000.

18. Mari JJ, Williams P. A comparison of the validity of two psychiatric screening questionnaires (GHQ12 and SRQ-20) in Brazil, using Relative Operating Characteristic (ROC) analysis. Psychol Med 1985; 15(3):651-659.

19. Gouveia VV, Chaves SSS, Oliveira ICP, Dias MR, Gouveia RSV, Andrade PR. A utilização do QSG-12 na população geral: estudo de sua validade de construto. Psicologia: Teoria e Pesquisa 2003; 19(3):241-248.

20. Harris PA, Taylor R, Thielke R, Payne J, Gonzalez N, Conde JG. Research electronic data capture (REDCap) - a metadata-driven methodology and workflow process for providing translational research informatics support. Journal of biomedical informatics 2009; 42(2):377-381.

21. Victora CG, Huttly SR, Fuchs SC, Olinto MT. The role of conceptual frameworks in epidemiological analysis: a hierarchical approach. Int J Epidemiol 1997; 26(1):224-227.

22. Santos EG, Siqueira MM. Prevalência dos transtornos mentais na população adulta brasileira\&58; uma revisão sistemática de 1997 a 2009. Jornal Brasileiro de Psiquiatria 2010; 59(3):238-246.

23. Ludermir AB, Schraiber LB, D'Oliveira AF, Franca-Junior I, Jansen HA. Violence against women by their intimate partner and common mental disorders. Soc Sci Med 2008; 66(4):1008-1018.

24. Parreira BDM, Goulart BF, Haas VJ, Silva SR, Santos Monteiro JC, Gomes-Sponholz FA. Transtorno mental comum e fatores associados: estudo com mulheres de uma área rural. Revista da Escola de Enfermagem da USP 2017; 51(3):1-8.

25. Araújo TM, Pinho PdS, Almeida MMG. Prevalência de transtornos mentais comuns em mulheres e sua relação com as características sociodemográficas e o trabalho doméstico. Revista Brasileira de Saúde Materno Infantil 2005; 5(3):337-348.

26. Hillemeier MM, Weisman CS, Chase GA, Dyer AM Mental health status among rural women of reproductive age: findings from the Central Pennsylvania Women's Health Study. Am J Public Health 2008; 98(7):1271-1279.

27. Shidhaye R, Patel V. Association of socio-economic, gender and health factors with common mental disorders in women: a population-based study of 5703 married rural women in India. Int J Epidemiol 2010; 39(6):1510-1521.

28. Ishida K, Stupp P, Serbanescu F, Tullo E. Perinatal risk for common mental disorders and suicidal ideation among women in Paraguay. Int J Gynaecol Obstet 2010; 110(3):235-240.

29. Pinho PS, Araújo TM. Associação entre sobrecarga doméstica e transtornos mentais comuns em mulheres. Revista Brasileira de Epidemiologia 2012; 15(3):560-572.

30. Prins H. Social Origins of Depression. A Study of Psychiatric Disorder in Women. New York: Free Press; 1978.
31. Badsiwal R. Rural Women and Marriage. Sociology and Criminology 2015; 3:2.

32. Fergusson DM, Horwood LJ, Boden JM. Abortion and mental health disorders: evidence from a 30-year longitudinal study. Br J Psychiatry 2008; 193(6):444-451.

33. Coleman PK. Abortion and mental health: quantitative synthesis and analysis of research published 19952009. Br J Psychiatry 2011; 199(3):180-186.

34. Piccinini CA, Gomes AG, Moreira LE, Lopes RS. Expectativas e sentimentos da gestante em relação ao seu bebê. Psicologia: Teoria e Pesquisa 2004; 20(3):223232.

35. Campo-Arias A, Herazo E, Barros-Bermúdez JA, Rueda-Jaimes GE, Díaz-Martínez LA. Common Mental Disorders in Colombian Women. Revista Colombiana de Psiquiatría 2011; 40(2):244-250.

36. Jacka FN, Maes M, Pasco JA, Williams LJ, Berk M. Nutrient intakes and the common mental disorders in women. J Affect Disord 2012; 141(1):79-85.

37. Patel V, Kirkwood BR, Pednekar S, Weiss H, Mabey D. Risk factors for common mental disorders in women. Population-based longitudinal study. Br J Psychiatry 2006; 189(6):547-555.

38. Koob GF, Le Moal M. Drug addiction, dysregulation of reward, and allostasis. Neuropsychopharmacology 2001; 24(2):97-129.

39. Juruena MF, Cleare AJ, Pariante CM. The hypothalamic pituitary adrenal axis, glucocorticoid receptor function and relevance to depression. Revista brasileira de psiquiatria 2004; 26(3):189-201.

40. Kendler KS, Neale MC, MacLean CJ, Heath AC, Eaves LJ, Kessler RC. Smoking and major depression: a causal analysis. Arch Gen Psychiatry 1993; 50(1):36-43.

41. Murthy SK, Shamasundar C, Prakash O, Prabhakar N. Psychiatric morbidity in general practice - a preliminary report. Indian Journal of Psychiatry 1981; 23(1):40-43.

42. Gureje O, Obikoya B. The GHQ-12 as a screening tool in a primary care setting. Soc Psychiatry Psychiatr Epidemiol 1990; 25(5):276-280.

43. Araya R, Wynn R, Lewis G. Comparison of two self administered psychiatric questionnaires (GHQ-12 and SRQ-20) in primary care in Chile. Soc Psychiatry Psychiatr Epidemiol 1992; 27(4):168-173.
Artigo apresentado em 13/08/2018

Aprovado em 18/11/2018

Versão final apresentada em 20/11/2018 
